\title{
SPORT, INSERTION, INTÉGRATION
}

\section{Patrick MIGNON}

(Article paru dans : Hommes et migrations, 2000, juillet-août, $\left.n^{\circ} 1226, p p .15-26\right)$

Dans sa fonction idéale, le sport est synonyme de justice et d'égalité des chances, de participation à la société, d'amélioration personnelle, de discipline - individuelle et collective, morale et physique. Il contribue ainsi à la transformation de l'individu en citoyen et crée des modèles, des figures exemplaires. Mais la réalité de la compétition et le culte de la victoire viennent s'opposer à ces valeurs: il serait d'ailleurs risqué d'affirmer que le sport permet une intégration facile. S'il est encore trop tôt pour évaluer, en France, son impact réel sur les jeunes issus de l'immigration, on connaît déjà les dangers d'une spécialisation précoce et, plus encore, d'une spécialisation qui serait liée à l'origine du sportif.

L'intégration est ce processus continu par lequel une société transcende les différences entre les populations vivant sur son territoire. Elle désigne les formes de participation à la société globale par l'activité professionnelle, l'apprentissage des normes de consommation matérielle, l'adoption des comportements familiaux et culturels (1). Institution de la société dont il exprime les valeurs, le sport participe aux mécanismes spontanés ou volontaires qui assurent sa reproduction. Par ses principes, ses règles, son fonctionnement, son appel à la discussion, il prend place dans ce processus continu d'intégration, dans la mesure où sa pratique implique, comme l'école ou le travail, la participation à un univers social distinct de la famille et signifie le mouvement d'appropriation progressive des différents univers qui définissent une société, Ce qui est valable pour la pratique l'est aussi pour le spectacle sportif, au sens où celui-ci suppose par exemple le déplacement dans l'espace urbain.

Très rapidement, on pense ici au rôle qu'a pu jouer le Tour de France avant 1914 dans la production d'un sentiment d'identité nationale, à travers la mise en scène d'un territoire amputé puis, après 1918, celle de la France des multiples terroirs. On pourra dire la même chose de l'organisation progressive des championnats nationaux dans les différentes disciplines, signifiant, grâce au stade nouvellement construit, le passage à la modernité et l'intégration des identités locales dans la nation française, à travers les cérémonies que sont les remises des trophées à la fin d'une saison ou la composition des équipes nationales. De ce point de vue, la France occupe une position originale en Europe puisque, très vite, elle intègre des athlètes venant aussi bien de son empire colonial que d'autres pays européens, réalisant dans le sport les principes d'une nationalité fondée sur le droit du sol. On peut ainsi considérer que les différents sports, en France, ont contribué à intégrer les fils des immigrations polonaise, italienne, espagnole puis maghrébine et africaine, à tous les niveaux de la pratique.

\section{VERS UNE POLITIQUE VOLONTARISTE}

Il n'en sera pas, bien sûr, de même dans tous les pays, car les différences se traduiront dans les formes d'accès à la citoyenneté entre, par exemple, droit du sol et droit du sang dans l'acquisition de la citoyenneté (2), mais aussi dans l'équilibre qui se constitue entre les différentes formes de représentation du peuple et des rapports entre appartenance communautaire et appartenance politique. Car le sport devient ainsi une des instances de socialisation où l'on apprend les valeurs de la collectivité, à travers la pratique et l'appartenance à une association. Il participe à l'intégration puisqu'il constitue l'une des pratiques par lesquelles on devient autre chose que le nouveau ou l'étranger, par la possibilité 
offerte de partager un loisir commun ou même de s'engager dans un processus de mobilité sociale grâce au professionnalisme. Cette intégration se produit à différents niveaux et à partir de différentes logiques. L'équipe nationale la manifeste à un autre niveau que le club local ou le club affilié aux organisations du sport ouvrier ou du sport catholique.

Les choses changent ou se compliquent à partir des années quatre-vingt, et le terme "intégration" signifie alors autant une politique volontariste qu'un processus spontané. La question explicite du sport comme facteur de socialisation et d'intégration n'est pas nouvelle. Même si le vocabulaire était un peu différent, la promotion du sport au XIXe siècle était liée à une interrogation sur la force de cohésion des sociétés nées de la Révolution française et de la révolution industrielle. Aujourd'hui, on accorde, dans les politiques publiques, une place considérable au sport parce qu'il apparait, avec la culture, comme l'autre moyen que l'on peut mettre à contribution pour réparer un tissu social qui se défait, pour retrouver les chemins de l'insertion économique, bref, pour faire face à la dérive des quartiers en difficulté, à l'échec scolaire, à la délinquance, aux risques de communautarisme et de racisme. La question des banlieues est au centre, et avec elle la question des jeunes issus de l'immigration - même si l'intégration concerne d'autres groupes comme les femmes, les retraités ou les handicapés dans la mesure où le sport est un aspect de l'égalité à atteindre, en plus du caractère formateur que représente l'expérience sportive. Il y a de fortes raisons à cette mobilisation du sport comme moyen de résoudre des problèmes sociaux: son attrait, non démenti depuis vingt ans, son rapport privilégié à la jeunesse - il est porteur de l'espoir d'un avenir différent qui échapperait au poids des déterminismes sociaux et culturels -, son histoire plus ancienne, surtout en France - où le sport est d'emblée lié à la capacité à faire participer tous les groupes habitant sur le territoire -, et enfin les valeurs qu'il incarne : l'égalité, la juste compétition et la visibilité de tous.

\section{LE SPORT SOCIALISATEUR ET INTÉGRATEUR ?}

On peut dessiner un parc:ours idéal de la socialisation et de l'intégration grâce au sport. C'est celui que l'on peut retracer par les références à l'histoire, par les enquêtes sociologiques ou ethnographiques, ou par les incitations et les projets identifiables dans les politiques publiques s'appuyant sur les valeurs du sport, telles que les ont définies Pierre de Coubertin et les Anglo-Saxons. On pourrait dire que le sport vaut parce qu'il est une société en miniature, et une société qui donne sa chance à chacun puisque toutes les caractéristiques physiques peuvent s'épanouir, grâce à une offre quasi universelle sur l'ensemble du territoire. Ainsi le sport est déjà un aspect de l'égalité et du droit de chacun à participer, selon ses moyens et sa volonté, aux activités et pratiques de la société. Chacun a le droit de pratiquer l'activité sportive qui lui convient. Comme l'école, le sport ne connaît pas d'obstacle de principe à la participation de tous, quelle que soit l'origine.

Au-delà de l'affirmation de principes, on établit l'intérêt socialisateur du sport à travers plusieurs aspects. Le premier élément est constitué par l'apprentissage des règles qu'il faut respecter pour pouvoir participer au jeu, ce qui implique la conscience de références extérieures qui déterminent l'activité. Ces règles sont personnifiées par l'arbitre pour le respect des règles de discipline, et par l'entraîneur pour celles de la logique propre à l'activité exercée, logique qui débouche sur un processus d'amélioration indéfinie de l'individu. C'est aussi un jeu dans lequel chacun découvre des possibilités motrices spécifiques, qu'il met enjeu avec d'autres. Dans un sport individuel comme dans un sport collectif, la pratique sportive renvoie à un apprentissage de la vie collective, que l'on parle de la nécessaire division des rôles sociaux ou de la découverte de soi à travers le contact avec les autres. Qu'il soit pratiqué dans 
un cadre officiel ou non, qu'il soit "nouveau" ou traditionnel, le sport contribue à la construction individuelle par la multiplication des expériences, laquelle favorise l'apprentissage de la négociation avec les autres et permet d'expérimenter ses propres limites.

Les limites sont celles qui sont fixées par l'individu lui-même, par sa socialisation familiale par exemple, et celles que fixent les règlements du sport pratiqué. Le sport en général constitue une reprise du principe égalitaire cité plus haut, il initie aux valeurs de justice et d'égalité, qu'il confronte à la réalité de l'inégalité et à la part de la chance et du hasard. Cette tension, le sport propose de la dénouer grâce au fair play : savoir gagner et perdre dans le respect de l'esprit du jeu est un bon exercice de maitrise de soi pour faire face à l'adversité, tout comme le contrôle de soi pendant l'affrontement sportif permet d'être en accord avec les règles et l'esprit du jeu. Le sport, normalement, fait comprendre qu'il s'agit d'un jeu.

\section{DE L'INDIVIDU AU CITOYEN}

Il y a ainsi dans le sport une discipline morale, mais aussi physique, une reconnaissance de soi qui le met en rapport avec d'autres normes. L'idée que le sport est bon pour la santé, qu'il suppose une forme de contrôle de son hygiène de vie, une maîtrise de soi, etc., signifie une intériorisation de normes morales et corporelles de la société française, ce qui bien évidemment suscite des résistances: les résistances à la pratique féminine du sport en constituent une bonne illustration. Globalement, il implique une autonomisation de l'individu, une meilleure connaissance de son corps, une plus grande liberté de mouvement. Toutes ces raisons montrent pourquoi on peut légitimement s'appuyer sur le sport dans une perspective de recherche d'intégration. En s'arrêtant là, on pourrait déjà décrire un individu ayant ouvert ses horizons, plus maître de lui-même et de son corps, inscrit dans un processus de mise à distance des appartenances.

Dans le schéma de l'intégration à la française, le sport contribue aussi à la transformation de l'individu en citoyen parce qu'il appartient au tissu associatif: le sportif est membre d'un club. Par le paiement de sa cotisation, par sa participation aux assemblées du club ou aux manifestations conviviales, le sportif inscrit son action dans le formalisme des gestes politiques - le vote - ou dans la convivialité qui mêle tous les âges et tous les statuts sociaux. Il exerce ainsi des responsabilités. En tant que vedette du club, quand bien même nous serions dans les plus modestes des niveaux du sport, il est reconnu, quelle que soit son origine, comme membre exemplaire de la collectivité.

En droit, il reste encore du chemin à parcourir. Les responsabilités sportives peuvent se transformer en responsabilités politiques - devenir élu - ou en responsabilités sociales, lorsque le pratiquant devient à son tour éducateur sportif ou animateur social pour aider d'autres jeunes à faire leur chemin dans la société. Le joueur professionnel ou l'éducateur sportif sont des figures exemplaires des possibilités offertes par le sport tant en matière de mobilité sociale, un des plus sûrs garants de l'intégration, que d'inscription dans les valeurs de la citoyenneté.

\section{L'INDISCUTABLE PRÉPONDÉRANCE DU FOOT}

Que savons-nous de la participation des jeunes de l'immigration dans le sport ? Pour des raisons que l'on connaît bien, on ne dispose pas d'enquêtes quantitatives qui permettraient de donner une description satisfaisante des pratiques et des goûts spécifiques de ces jeunes. 
Sans doute peut-on dire qu'ils ne sont pas très différents de ceux de l'ensemble de la population juvénile: le sport fait très certainement partie de leurs activités favorites (3). Il serait particulièrement intéressant de savoir si, comme pour l'ensemble de la population, ces pratiques sont tout autant tributaires du sexe - les filles pratiquant de façon générale moins que les garçons, la question étant dans quelle proportion en ce qui concerne les jeunes filles issues de l'immigration - ou de l'appartenance sociale. En effet, les enfants des classes populaires pratiquent moins que les enfants de cadres ou des professions intermédiaires, et on ignore si dans le cas des jeunes des quartiers, les espérances de promotion par le sport viendraient corriger ce constat.

Toutefois, à travers les visites dans les clubs, à l'Insep ou sur les terrains improvisés, ou par les images que les médias donnent des différents sports, on peut établir une liste des sports selon qu'ils attirent plus ou moins les jeunes issus de l'immigration. Le football, par exemple, est incontestablement le sport le plus populaire, pour le coup sans doute le vrai sport universel, que ce soit par goût, parce qu'il est accessible partout, ou par l'idée d'une promotion sociale que les succès d'un Zidane ou d'un Vieira entretiennent. Cette popularité se retrouve aussi bien dans les équipes professionnelles des trois divisions que dans les petits clubs un peu partout en France. On a bien sûr aussi en tête l'image des play-grounds de basket, qui nous renvoie au modèle américain et à la quasi-domination des Afro-Américains sur ce sport aux États-Unis, et au modèle de réussite sportive et sociale d'un groupe dominé, avec des stars comme Michael Jordan et ses "équivalents" français comme Sonko ou Tariq Abdul Wahad. En termes de pratique, on est toutefois très loin du football, malgré les volontés fortes, affichées durant les années quatre-vingt-dix par des sponsors comme Nike, de développer le basket en France.

\section{GAINS RAPIDES CONTRE MOBILITÉ SOCIALE}

À un niveau quantitatif encore moindre, on relèvera l'existence de clubs d'athlétisme axés principalement sur les courses de demi-fond ou sur le cross, où sont présents des jeunes issus de l'immigration maghrébine, tandis que l'on retrouvera dans d'autres clubs des jeunes d'origine africaine. On peut y voir le maintien d'une longue tradition qui remonte à Alain Mimoun et aux autres coureurs d'origine algérienne ou marocaine qui se sont distingués en France, et aux succès présents des athlètes maghrébins ou africains. Toutefois, comme pour le football, on ne sait pas s'il s'agit d'une pente "naturelle" ou d'un recrutement des clubs sur le marché international, au regard du succès des Noirs ou des Maghrébins dans les grandes compétitions. Bien sûr, quand on pense aux jeunes des quartiers, on pense inévitablement aux sports de combat et aux arts martiaux sous toutes leurs formes, des sports reconnus officiellement comme la boxe, la boxe française, la lutte, sans compter les différentes boxes pied-poing (kick boxing, boxe thaï). Là encore, les chiffres manquent mais on sait que certains de ces sports, tous ceux relevant de la boxe notamment, sont traditionnellement, et dans tous les pays, les sports des derniers arrivants et des plus pauvres.

Qui pratique en effet ces différents sports en France ? La visite de quelques clubs et les témoignages des éducateurs nous disent que ce sont des jeunes (de quinze à trente ans) d'origine africaine, maghrébine, des Gitans, les enfants des mineurs du Nord et de l'Est, les nouveaux arrivants des pays de l'Est. On peut y voir, pour certains, une liaison incontestable avec le mode de vie, la rue et ses valeurs, et la place du combat parmi ces valeurs; ce pourrait être un effet de l'offre sportive; on peut aussi y voir l'attrait de sports qui promettent des gains rapides, à défaut d'une véritable mobilité sociale. Cette mobilité sociale, on pourrait aussi en évaluer les effets, non seulement en termes de notoriété médiatique de certaines stars ou de 
nombre de joueurs professionnels, mais aussi à partir de la présence, parmi les cadres et éducateurs des clubs sportifs, des jeunes et maintenant moins jeunes des quartiers. Pour l'instant, on ne peut que remarquer la présence conséquente d'entraîneurs de football, ou de moniteurs et professeurs dans les sports de combat, qui ont transformé une carrière sportive en une profession d'encadrement ou de responsabilité.

\section{DANS LES STADES, UN PUBLIC PLUS REPRÉSENTATIF}

À côté des succès incontestables, on voit bien que d'autres sports importants dans l'imaginaire français sont absents: c'est le cas du cyclisme, qui n'importe comment éprouve des difficultés à attirer les jeunes de façon générale, ou du rugby, où ne brillent pour l'instant que quelques stars comme Benazzi, mais vers lequel quelques clubs toutefois, et pas seulement en région parisienne, ont semble-t-il réussi à attirer des jeunes de l'immigration. Mais on touche là un problème plus général sur lequel nous reviendrons, à savoir que les sports recrutent dans des milieux sociaux différents selon la place qu'ils occupent dans la stratification sociale: le rugby, le judo - avec toutefois les réussites que l'on connaît des judokas d'origine maghrébine -, à plus forte raison l'escrime, et à plus forte raison encore le golf ou l'équitation vont de pair avec l'appartenance à des milieux de mieux en mieux dotés en capital économique et en capital culturel. De ce point de vue, la distribution des sports parmi les jeunes des banlieues renvoie à leur appartenance aux classes populaires, et le palmarès des sports n'est pas très surprenant.

Si l'on veut évoquer la participation au sport, il faut aussi envisager la participation au spectacle sportif. De ce point de vue, outre les images des rues de France après la Coupe du monde de 1998, il convient d'être attentif aux transformations des publics des stades. Ainsi, on peut noter que le public du Parc des Princes, et c'est là un lieu sensible quand on veut parler des problèmes de racisme, s'est considérablement modifié en quelques années. Il y a dix ans, on pouvait considérer que le stade, à part les représentants des immigrations portugaises ou espagnoles ou de la population antillaise, n'offrait pas une image représentative de la population du grand Paris. Ce n'est plus tout à fait le cas aujourd'hui, alors que l'on voit plus fréquemment les "jeunes" - les 16-40 ans - issus de l'immigration fréquenter ce stade, certains participant même à des groupes ultra (c'est encore plus évident à Marseille). Sont-ce tous ces cadres sportifs qui viennent voir du football de haut niveau ? Sont-ce, comme une bonne partie des spectateurs dans les stades, les représentants de cette partie des populations issues de l'immigration qui est en plein mouvement de mobilité sociale ascendante ? Pour les plus jeunes, il faut toutefois faire la part des initiatives volontaristes des maires ou des conseils généraux, et donc de l'engagement pour le Paris-Saint-Germain et/ou pour le match de football comme loisir de prédilection. Mais on notera que le maillot du PSG se porte plus volontiers maintenant sur les terrains officiels ou improvisés des banlieues, et qu'il n'est plus seulement le signe de ralliement des "petits blancs" comme il l'était dans les années quatrevingt.

\section{UNE CERTAINE MÉFIANCE DES ADOLESCENTS}

On voit bien que les réponses ne sont pas pleinement satisfaisantes car on manque de véritables enquêtes, ethnographiques ou statistiques, pour donner des éléments fiables. De la même manière, on manque d'analyses sur les rapports qu'entretiennent les différentes cultures de l'immigration avec l'engagement dans le sport. Ainsi, un entraîneur d'origine algérienne me faisait remarquer qu'il avait plus de mal à garder ses "Arabes", qui avaient tendance à déserter les entraînements puis l'équipe autour de l'âge de quinze ans, que ses "Blacks", plus motivés 
semble-t-il pour continuer. Est-ce local ? Est-ce un effet de la grande proximité des jeunes "Beurs" avec les autres jeunes de leur âge ? Est-ce un effet des politiques de recrutement des clubs ? Et puis, il y a encore cette grande question de "l'effet Zidane" ou de "l'effet Coupe du monde". On en a vu très vite les résultats dans les clubs de football, mais pour le reste les choses sont un peu plus difficiles à évaluer. Dans une société où les jeunes sont aussi pleinement intégrés à la société par leur participation à la culture médiatique, on n'est jamais sûr que les valeurs de citoyenneté dont pouvait être porteur l'événement, ne sont pas largement contrebalancées par celles de la réussite économique à tout prix.

Mais qu'est-ce qui empêche que les choses fonctionnent aussi bien ? Certaines réalités montrent qu'il faut manier avec précaution l'idée d'une intégration facile par le sport; il ne faut pas lui attribuer plus de pouvoir qu'il ne peut en avoir. Ainsi, l'apprentissage de la citoyenneté peut être contrarié par le mouvement de fuite des clubs. C'est un constat fait depuis plusieurs années: les associations ont du mal à garder leurs jeunes éléments après l'âge de quatorze ou quinze ans. Cela est tout d'abord dû au mouvement d'autonomisation de la jeunesse, qui rend les adolescents méfiants vis-à-vis des formes d'engagement et les incite à créer par euxmêmes leurs lieux de socialisation. Les jeunes de l'immigration sont jeunes, et à ce titre, ils sont aussi "à côté" et peut-être un peu plus que ne le sont les jeunes des classes populaires.

Sans doute peut-on aussi renvoyer cette désaffection à l'absence de démocratie dans les clubs, à leur caractère trop étroitement sportif et moins citoyen qu'il ne devrait l'être, car finalement, ce que l'on veut quand on est adolescent, c'est être reconnu, pas seulement comme un athlète potentiel mais aussi comme un individu porteur de particularités. Le racisme n'est alors pas toujours loin. Ce peut être aussi la pauvreté des clubs et de l'offre qu'ils sont en mesure de proposer et qui, après la Coupe du monde de football, a découragé beaucoup de jeunes garçons et de jeunes filles. Comme peuvent les décourager les formes ordinaires et extraordinaires du racisme, que l'on pense aux manifestations de certains supporters du PSG, aux réactions des publics des petites villes quand viennent des équipes de banlieues parisiennes ou marseillaises.

\section{DE LA TENSION ENTRE IDÉAL ET RÉALITÉ}

L'un des problèmes du sport est que sa force intégratrice repose aussi en partie sur la valorisation du collectif. À ce titre, il induit que le monde des spectateurs est divisé entre "eux" et "nous". Certains supporters des clubs de football refusent que des "étrangers" soutiennent aussi le club. De la même manière, les incidents autour des terrains de football, un peu partout en France, découlent aussi de cette conception des territoires, de l'opposition entre "eux" et "nous" et d'une socialisation incomplète. Cela s'explique en partie par le fait que les clubs ont du mal à intégrer à la fois une logique sportive et une logique plus sociale ou de reconnaissance de la diversité des attentes vis-à-vis du sport. Aujourd'hui, dans les discussions sur les clubs communautaires, on devrait sans doute prendre en compte en quoi ces clubs relèvent d'une logique discutable du communautarisme, mais aussi en quoi ils sont l'expression de la difficulté des institutions sportives à équilibrer, ce qui a longtemps été leur mission, logique sportive et logique explicitement civique.

On a donc souvent raison d'opposer les idéaux du sport à sa réalité, et d'évoquer en quoi le développement de la compétition est contraire aux valeurs qu'il prétend préserver. Le sport est lui-même plongé dans la société: il défend des valeurs comme la victoire ou la compétition, qui apparaissent comme des enjeux politiques, sociaux ou économiques. À ce titre, les règles peuvent être relativisées par la victoire à tout prix, qui institutionnalise la 
triche, la pression sur les arbitres et sur les joueurs, donc la violence. Dans ce cas de figure, l'éducateur disparaît, les valeurs du sport et leur caractère exemplaire avec lui.

\section{VRAIE OU FAUSSE INTÉGRATION?}

L'intégration par le sport rencontre aussi ses limites dans les évolutions actuelles du sport. Tous les sportifs ne sont pas des athlètes de haut niveau, professionnels ou susceptibles de participer aux Jeux Olympiques. Ce qui signifie que les investissements dans le sport sont coûteux, moins en termes de matériel (les sports les plus populaires sont les moins coûteux financièrement) mais en termes d'investissement social et psychologique. Le risque est de penser que le sport permet l'ascension sociale que l'école ne paraît pas garantir, ou permet de faire l'économie de l'école quand les places au sommet sont rares. Dans ce cas, au lieu d'être l'une des étapes du cheminement social et de la construction d'une multiplicité de liens, il expose le sportif aux inconvénients de la spécialisation précoce, ou à l'individualisme exacerbé de certains pratiquants. Le sport en tant qu'activité exclusive est une activité qui enferme dans un petit monde dont il faut pouvoir sortir. Les problèmes de reconversion se posent quand l'engagement dans l'activité sportive signifie l'abandon d'autres voies de socialisation et d'intégration. À un certain moment, cette intégration fonctionne, mais l'échec sportif ou l'accident viennent reposer le problème quelques années plus tard. L'autre risque est la réalité du marché économique du sport, qui peut entretenir de fausses espérances.

Enfin, un dernier risque existe, celui de la spécialisation culturelle dans le sport. On peut ici se référer à l'exemple américain. Les Noirs américains connaissent-ils une fausse ou vraie intégration par le sport ? Que se passe-t-il quand on incite à faire du sport ? Peut-être la même chose que lorsque l'on oriente les jeunes filles vers la coiffure. L'attrait pour l'activité sportive existe mais il faut garder à l'esprit que le sport reste un secteur dominé, moins valorisé et fragile en terme d'intégration. Ne risque-t-il pas, au nom de l'attrait et des qualités "naturelles" des jeunes des banlieues, d'assigner certains groupes à ce qui n'est plus alors une vocation, mais la seule voie de promotion proposée ? La question des liens entre sport et jeunes issus de l'immigration est donc encore largement à travailler.

\section{Notes}

1- Je m'appuie sur les définitions données par D. Schnapper, La France de l'intégration. Sociologie de la nation en 1990, Gallimard, Paris, 1991.

2- Sur tous ces points, voir l'ouvrage de P. Lanfranchi et A. Wahl, Les footballeurs professionnels en France, Hachette, 1996

3- Une enquête est en cours sur les pratiques sportives des français (Ministère de la Jeunesse et des Sports et INSEP), qui apportera des éléments chiffrés. Elle sera prolongée par une étude sur les pratiques sportives des jeunes. 